\section{Structured contraceptive counseling with shared decision making: Effects of implementation at a university student health and wellness clinic}

Judith S. Wackenhut, DNP, PNP-C; Amanda L. Ellis, DNP, AGNP-C; Kathleen H. Pridgen, MD; and Allison Shorten, PhD, RN, FACM, FAAN

or the first time in decades, the United States has reported a significant decline in the incidence of unintended pregnancies. ' Using data from the National Survey on Family Health, the overall unintended pregnancy rate is currently calculated at $45 \%$. ${ }^{1}$ Women in their 20 s experience the highest occurrence of unintended pregnancy, accounting for $55 \%$ of all cases. ${ }^{2}$ Over half of the 18 million students attending US colleges are women in this age group, making college campuses a critical setting for providing education about contraception. ${ }^{3}$ In a survey of community college students, participants often perceived their risk of pregnancy as low and overestimated their method's effectiveness, even when using it incorrectly. ${ }^{4}$ The 2015 National College Health Assessment found that $65 \%$ of college students reported

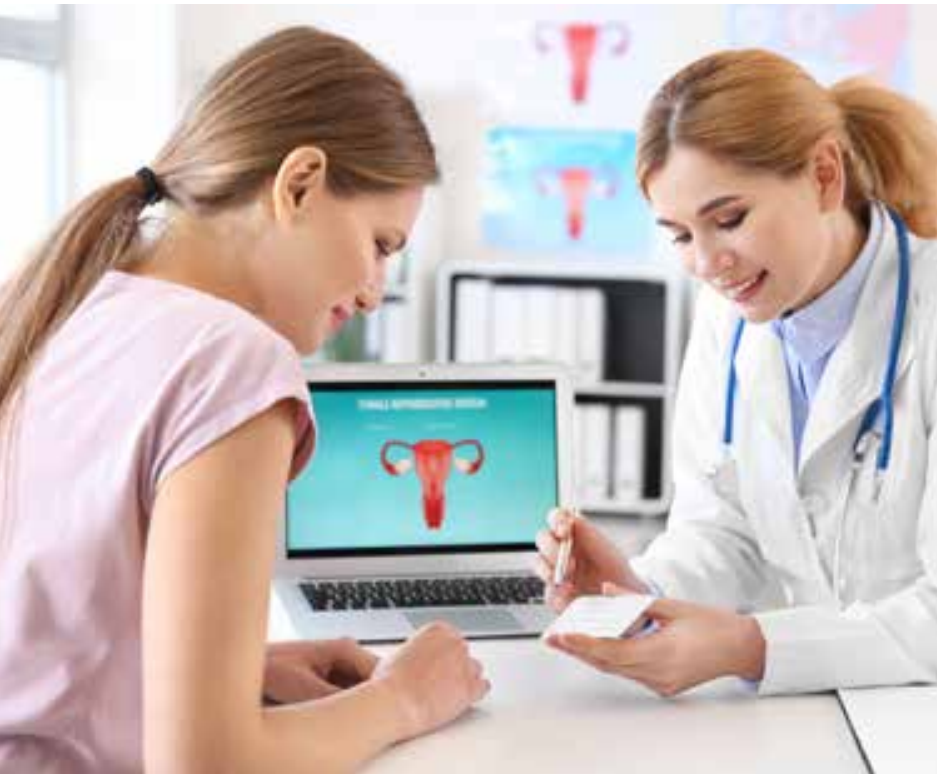

being sexually active in the past 12 months. Of those having sexual intercourse in the past 30 days, only $50.1 \%$ reported using any form of contraception. ${ }^{5}$

Enhancing the quality and consistency of contraceptive counseling is considered a key component of efforts to prevent unintended pregnancy. ${ }^{6}$ College healthcare visits provide an ideal opportunity for contraceptive education, and healthcare providers in these settings play a crucial role in delivering such services.

Contraceptive counseling has moved away from clinician-directed counseling to a patient-centered approach that keeps the patient's wishes and preferences at the forefront and has been shown to increase contraceptive continuation and satisfaction. ${ }^{7}$ Shared decision making (SDM) is recommended for value-sensitive healthcare decisions, such as choosing a method of contraception. Women must weigh the costs and benefits associated with their options and align personal values and needs to make a choice that is best for them and to which they can adhere. ${ }^{8}$ With SDM, providers share evidence-based information and use values clarification to help women align their choices with individual preferences. ${ }^{9}$ SDM requires clear steps: establish rapport and build trust, seek the woman's perspective, demonstrate caring/concern, and invest in the shared enacted decision. ${ }^{10}$ Structured contraceptive counseling (SCC) that utilizes both audio and visual aids to provide information and to engage the patient in dialogue facilitates making an informed, medically appropriate contraceptive choice. Using both SCC and SDM may improve the quality of contraceptive counseling and ultimately increase contraceptive uptake and adherence.

\section{Purpose}

The goal of this project was to design and implement a contraceptive counseling protocol using an SDM framework, embedded within the flow of care provided in a university health clinic. The purpose was to educate staff about SCC with SDM, provide improved patient contraceptive education resources, and increase both patient and provider satisfaction with the counseling process.

\section{Methods}

The university's institutional review board determined the project to be quality improvement, thus not requiring approval for human subjects' research. The setting was a mid-size, public state-based research university student health and wellness center. All staff (six nurse practitioners and three physicians) agreed to participate.

This project involved development and implementation 


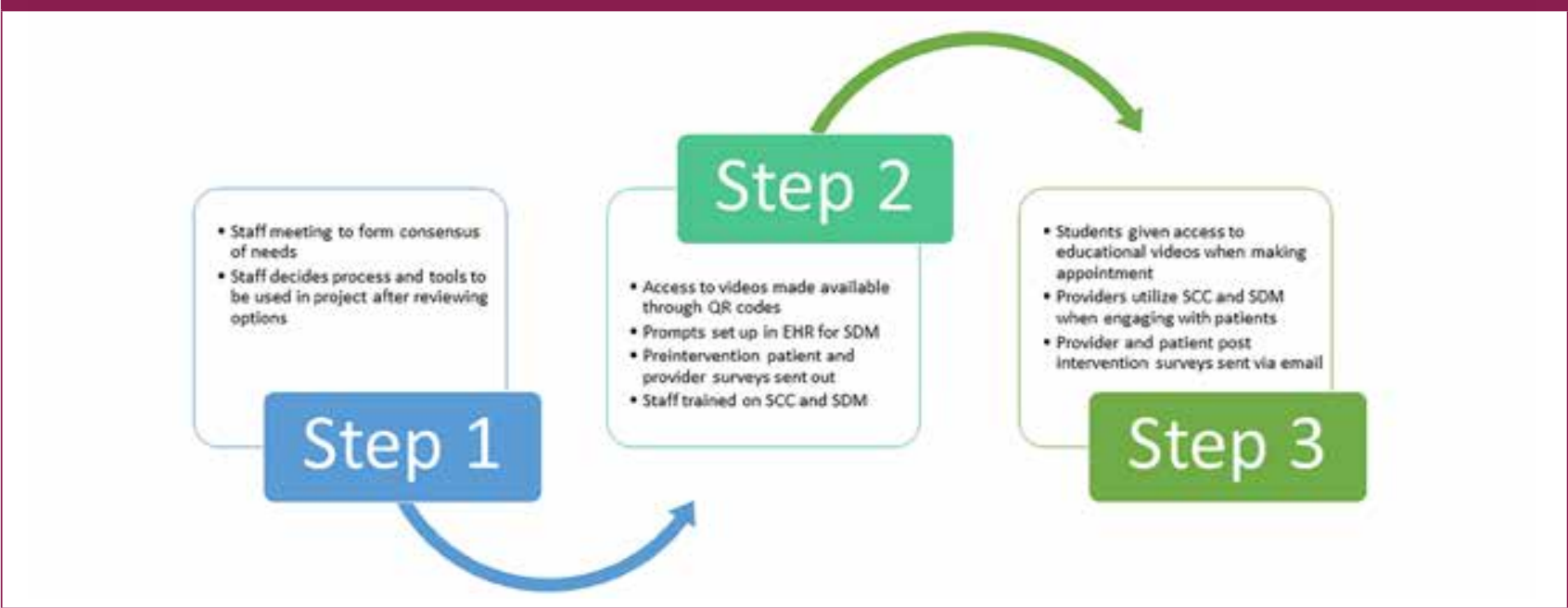

EHR, electronic medical record; SCC, structured contraceptive counseling; SDM, shared decision making.

of a new model of contraceptive counseling within the existing healthcare structure of the student health center. The need for this project was identified at staff meetings where gaps in the counseling process were acknowledged and a consensus was reached to develop and institute an evidence-based approach to providing counseling. Prior to this project, the approach to contraceptive care was based on provider preference, occurring with or without counseling from the provider, and did not necessarily include teaching aids or standardized information. A participatory approach and the three-step process were used (Figure). At the initial meeting, led by the project manager, options for counseling methodology were reviewed. A new care protocol for contraceptive counseling was generated using the United States Selected Practice Recommendations for Contraceptive Use." Group consensus was reached to offer patients access to educational resources prior to their appointment via quadratic residue codes to three contraceptive videos made available from the University of California San Francisco (UCSF) Bixby Center. The UCSF Bixby Center website titled "Beyond the Pill" includes three videos: Birth Control for Your Life, Birth Control that Really Works, and Hello Beautiful. ${ }^{12}$ Prompts for providers were entered into the electronic medical record to support and guide the SDM process. For the counseling session, providers selected the Bixby Center's "How Well Does Birth Control Work" chart, which shows contraceptive options by tiers of effectiveness. When necessary, providers could use the Centers for Disease Control and Prevention medical eligibility chart to explain possible contraindications for a requested method.

Staff were trained in SCC and SDM, focusing on the counseling tools and the SDM process. Providers agreed to document whether or not SCC was employed in the patient's health record. Patients were asked to complete a 10-question, online, anonymous patient experience survey, adapted with permission from the Contraceptive Action Plan's website resources page. ${ }^{13}$ Providers were asked to complete a monthly anonymous survey based on their recent experience with contraceptive counseling and their satisfaction with the process. The survey consisted of 10 questions that explored provider experience with patients during the implementation phase. All data for both surveys were collected via Survey Monkey and entered into SPSS software for analysis.

\section{Outcomes}

One month prior to implementation of the new model, a student satisfaction survey was emailed to patients who had been seen for contraceptive services over the prior 12 months. A total of 131 students completed the survey for baseline assessment. Student and provider surveys were sent out monthly. During the 3 months of project implementation, 53 students completed the satisfaction survey. Seven satisfaction questions showed improvement from baseline. One question relating to whether "enough information was received during visit" reached statistical significance $(P=.031)$. Similar results were observed for the provider satisfaction survey with an improvement from baseline to post intervention on all questions. All six providers participated in the surveys at each time interval. Only"educational pieces make the counseling process easier" reached statistical significance $(P=.034)$. 
Table. Free educational materials for practice, providers, and patients

\begin{tabular}{|c|c|c|}
\hline Name & Website & Content \\
\hline $\mathrm{CDC}$ & $\begin{array}{l}\text { cdc.gov/reproductivehealth/contraception/contraception } \\
\text { guidance.htm }\end{array}$ & $\begin{array}{l}\text { Practice guidelines, patient education materials, and links to } \\
\text { other resources }\end{array}$ \\
\hline USCF Bixby Center & beyondthepill.ucsf.edu/online-training $B$ & Training, education, tools, and materials \\
\hline Contraceptive Action Plan & contraceptiveactionplan.org/C & $\begin{array}{l}\text { Training, educational resources, e-learning for staff, } \\
\text { process procedures }\end{array}$ \\
\hline My Birth Control & mybirthcontrolapp.org/D & Patient education \\
\hline My Healthfinder & $\begin{array}{l}\text { health.gov/myhealthfinder/topics/everyday-healthy-living/ } \\
\text { sexual-health/choose-right-birth-control\#panel-1E }\end{array}$ & Patient education \\
\hline FPNTC & fpntc.org/training-packages/contraceptive-services $F$ & $\begin{array}{l}\text { Training, educational resources, e-learning for staff, } \\
\text { process procedures }\end{array}$ \\
\hline
\end{tabular}

CDC, Centers for Disease Control and Prevention; FPNTC, Family Planning National Training Center; USCF, University of California San Francisco.

\section{Limitations}

Limitations include the short time interval of data collection. There was also a high number of provider turnover during the implementation phase, which may have limited the effectiveness of the training the provider received during step 2 of the project.

\section{Implications}

Results of both patient and provider surveys done during this contraceptive counseling quality improvement project demonstrated that comprehensive patient-centered contraceptive counseling is possible in a busy college health clinic. Survey results showed the feasibility of using SCC with SDM and achieving both patient and provider satisfaction. The steps taken to improve the quality of counseling while maintaining clinic workflow can be implemented in any primary care setting. Free evidence-based counseling aids are readily available as well as online tools for staff training (Table). As demands grow for clinicians to increase productivity while providing quality care, finding a model to provide efficient yet evidence-based, client-centered, comprehensive contraceptive services is necessary. The next step in this university clinic site is to develop and implement counseling services that are available to and focus on contraceptive services for men, including increasing condom use. The development of future actions reflects an ongoing commitment to continuous quality improvement for patients seeking contraceptive services.

\section{Acknowledgments}

The project team would like to thank the staff and patients for their support and participation in this project. We are indebted to Dr. Kathleen Pridgen for her clinical mentorship during the project. The team also expresses our sincere gratitude to Dr. Allison Shorten for the dedication, wisdom, and patience she offered our team.

Judith S. Wackenhut is a pediatric nurse practitioner in private practice. Amanda L. Ellis is a nurse practitioner at Southeast Urogynecology in Jackson, Mississippi. Nicole $\mathrm{H}$. Metcalf is a family nurse practitioner at the University of Alabama Student Health and Wellness Center and adjunct instructor at the University of Alabama School of Nursing, Birmingham. Kathleen H. Pridgen is Associate Medical Director at the University of Alabama Student Health and Wellness Center, Birmingham. Allison Shorten is Professor and Department Chair of acute, chronic, and continuing care at the University of Alabama School of Nursing and Director of the Office of Interprofessional Curriculum, Center for Interprofessional Education and Simulation, at the University of Alabama at Birmingham. Judith S. Wackenhut discloses that she is employed at TherapeuticsMD. The other authors have no actual or potential conflicts of interest in relation to the contents of this article.

\section{References}

1. Finer LB, Zolna MR. Declines in unintended pregnancy in the United States, 2008-2011. NEngl J Med. 2016;374(9):843-852.

2. Kornides ML, Kitsantas B, Lindley LL Wu H. Factors associated with young adults' pregnancy likelihood. JMidwifery Womens Health. 2015;60(2):158-168.

3. Lehan Mackin M, Clark MK, McCarthy AM, Farris K. Knowledge and use of emergency contraception in college women. West J Nurs Res. 2015;37(4):462-480.

4. Coor A, Esie P, Dittus PJ, et al. Assessment of sexual health services at U.S. colleges and universities, 2001 and 2014. Sex Health. 2018;15(5):420-423.

5. American College Health Association. American College Health Association-National College Health Assessment II: Reference 
Group Executive Summary, Fall 2015. Hanover, MD: American College Health Association; 2016.

6. Dehlendorf C, Kimport K, Levy K, Steinauer JA. A qualitative analysis of approaches to contraceptive counseling. Perspect Sex Reprod Health. 2014;46(4):233-240.

7. Dehlendorf C, Fitzpatrick J, Steinauer J, et al. Development and field testing of a decision support tool to facilitate shared decision making in contraceptive counseling. Patient Educ Couns. 2017;100(7)1374-1381.

8. Elwyn G, Dehlendorf C, Epstein RM, et al. Shareddecision making and motivational interviewing: achieving patientcentered care across the spectrum of health care problems [published correction Ann Fam Med. 2014;12(4):301]. Ann Fam Med. 2014;12(3):270-275.

9. Frankel RM, Stein T. Getting the most out of the clinical encounter: the four habits model. JMed Pract Manage. 2001;16(4):184-191.

10. Truglio-Londrigan M, Slyer JT. Shared decision-making for nursing practice: an integrative review. Open Nurs J. 2018;12:1-14. health. Appendix A: Summary Chart of U.S. Medical Eligibility Criteria for Contraceptive Use, 2016. June 28, 2017. https:// www.cdc.gov/reproductivehealth/ contraception/mmwr/spr/appendixa.html.

12. Bixby Center for Global Reproductive Health. Bixby resources. Birth control for your life. 2018. https://bixbycenter.ucsf.edu/ bixby-resources\#contraceptive.

13. Contraceptive Action Plan. Patient Experience Survey. 2020. https://www.contraceptiveactionplan.org/index.php/tools-andresources-menu-item/patient-experience- survey-in-english.

\section{Web resources}
A. cdc.gov/reproductivehealth/contraception/contraception_ guidance.htm
B. beyondthepill.ucsf.edu/online-training
C. contraceptiveactionplan.org/
D. mybirthcontrolapp.org/
E. health.gov/myhealthfinder/topics/everyday-healthy-living/ sexual-health/choose-right-birth-control\#panel-1
F. fpntc.org/training-packages/contraceptive-services

11. Centers for Disease Control and Prevention. Reproductive

\section{(continued from page 14)}

cleanser along with other measures to prevent/control flares (Table 3). Patients benefit from education that guides them through avoiding the gauntlet of skin care products with unfounded advertisements promising a cure for acne.

\section{Lifestyle modifications}

It is postulated that foods with high glycemic index, dairy products, high stress levels, and lack of sleep may all play a role in the severity of acne in some females. ${ }^{10}$ Further studies are required to fully understand the impact of these factors, but talking to patients about how some of these can contribute may prompt lifestyle changes that may make a positive difference in both their acne and overall health.

\section{Conclusion}

Adult females may present with the common characteristics of acne vulgaris, but there are several unique etiopathogenic factors that make management of adult female acne more challenging. Cost, resources, potential for side effects, and patient preferences are important variables and the key to patient adherence. This article serves as a guide on recommendations for best evidence-based clinical practices and therapeutic decisions to help manage both the physical and psychological symptoms that often accompany it.

\section{References}

1. Rocha MA, Bagatin E. Adult-onset acne: prevalence, impact, and management challenges. Clin Cosmet Investig Dermatol. 2018;11:59-69.

2. Bagatin E, Freitas THP, Rivitti-Machado MC, et al. Adult female acne: a guide to clinical practice. An Bras Dermatol, 2019;94(1):62-75.

3. Dréno B, Thiboutot D, Layton AM, et al; Global Alliance to Improve Outcomes in Acne. Large-scale international study enhances understanding of an emerging acne population: adult females. J Eur Acad Dermatol Venereol. 2015;29(6):1096-1106.

4. Barros B, Thiboutot D. Hormonal therapies for acne. Clin Dermatol. 2017;35(2):168-172.

5. Callender VD, Alexis AF, Daniels SR, et al. Racial differences in clinical characteristics, perceptions and behaviors, and psychosocial impact of adult female acne. J Clin Aesthet Dermatol. 2014;7(7):19-31.

6. Zaenglein AL, Pathy AL, Schlosser BJ, et al. Guidelines of care for the management of acne vulgaris. J Am Acad Dermatol. 2016;74(5):945-973.e33.

7. Rosette C, Agan FJ, Mazzetti A, et al. Cortexolone $17 \alpha$-propionate (clascoterone) is a novel androgen receptor antagonist that inhibits production of lipids and inflammatory cytokines from sebocytes in vitro. J Drugs Dermatol. 2019;18(5):412-418.

8. Kim GK, Del Rosso JQ. Oral spironolactone in post-teenage female patients with acne vulgaris: practical considerations for the clinician based on current data and clinical experience. J Clin Aesthet Dermatol. 2012:5(3):37-50.

9. Bashir A, Lambert P. Microbiological study of used cosmetic products: highlighting possible impact on consumer health. J Appl Microbiol. 2020;128(2):598-605.

10. Bansal P, Sardana K, Vats G, et al. A prospective study examining trigger factors and hormonal abnormalities in adult female acne. Indian Dermatol Online J. 2020;11(4):544-550.

Web resource

A. npwh.org/courses/home/details/1621 